\title{
Stell Dir vor, es ist Ärztekammer ...
}

... und niemand weiss es. Ein absurder Gedanke? Wir werden darauf zurückkommen. Zunächst einige Gedanken anlässlich der vor knapp einem Monat durchgeführten Tagung des «Ärzteparlaments», deren ausführliches Protokoll am Anfang dieser Nummer zu finden ist.

Für den nicht direkt involvierten Beobachter war es auch an der letzten Ärztekammer eindrücklich, zu verfolgen, mit welchem Engagement, mit wieviel «verve» und Hartnäckigkeit um bestimmte Positionen gerungen wurde. Zwar zeigte sich verschiedentlich, dass Ärztinnen und Ärzte auch «nur» Politiker sind, das heisst, ein breites taktisches Instrumentarium bei Bedarf virtuos einsetzen. Doch hatte man von wenigen Ausnahmen abgesehen den Eindruck, dass «die Sache» gegenüber persönlichen Motiven klar im Vordergrund stand und die übergeordneten Interessen der gesamten Ärzteschaft nie aus dem Auge verloren wurden. Diese gemeinsame Grundhaltung ermöglichte es, bei aller Heterogenität immer wieder zu gemeinsam getragenen Lösungen zu kommen. Fast lehrbuchmässig liess sich dies bei einer Reihe von Anträgen beobachten, die nach Zugeständnissen von beiden Polen in einem Kompromiss endeten. Ernsthafte, konsensorientierte politische Arbeit, wie man sie im nationalen Parlament leider öfters vermisst.

Möglicherweise wird diese Haltung von der Erkenntnis der involvierten Akteure getragen, dass eine Ärzteschaft, die zwar «innenpolitische» Auseinandersetzungen nicht scheut, gegen «aussen» aber geschlossen auftritt, angesichts der ökonomischen und politischen Entwicklungen im Gesundheitswesen wichtiger ist denn je. Was für die Grundversorger gilt, müsste für die Ärzteschaft insgesamt erst recht gelten: Geeintes, entschlossenes Auftreten macht nicht nur Eindruck, sondern ist Voraussetzung für die Mitgestaltung der Bereiche, die das ärztliche Arbeitsfeld betreffen.
Geschlossenheit in diesem Sinne müsste auch ein starkes verbandspolitisches Bewusstsein der Basis einschliessen - die Ärztekammer ist in diesem Sinne lediglich die Spitze des Eisbergs. Wie steht es nun damit? Die Grundversorger, häufig «Kleinunternehmer» mit hoher Eigenverantwortung, haben ein solches Bewusstsein am 1. April eindrücklich demonstriert. Bei den jüngeren Kolleginnen und Kollegen in Weiterbildung präsentiert sich die Situation möglicherweise (noch) etwas anders. Im Hinblick auf die Ärztekammer habe ich aus Neugier völlig willkürlich einige Assistenzärztinnen und -ärzte aus meinem Bekanntenkreis darauf angesprochen, was sie über diese Institution wüssten. Das Ergebnis war ernüchternd. In einem Fall war nicht einmal der Begriff «Ärztekammer» bekannt, die übrigen hatten mehr oder weniger nebulöse Vorstellungen von der Funktion dieses Gremiums. Über die Statutenrevision wusste niemand Bescheid. Natürlich muss nun der Hinweis auf die mangelnde Repräsentativität einer solchen Stichprobe erfolgen. Und doch bleibt das Gefühl einer Kluft zwischen «Basis» und «Überbau». Diese ist aus meiner Sicht weder der einen noch der anderen Seite «anzulasten», sondern dürfte vorwiegend damit zusammenhängen, dass für Ärztinnen und Ärzte zu Beginn ihrer Karriere die Bedeutung verbandspolitischer Fragen keineswegs offensichtlich ist.

Wie könnte hier Abhilfe geschaffen werden? Müsste Verbandskunde in die Aus-, Weiter- und Fortbildung integriert werden wie Staatskunde in den Schulunterricht? Handlungsbedarf scheint jedenfalls gegeben - Ideen sind gefragt!

Bruno Kesseli 